\title{
Allergie-Kongress 2004, Aachen
}

\section{Wenn die neue Hüfte plötzlich Probleme macht ...}

\section{Das Einsetzen künstlicher Gelenke ist in der Orthopädie inzwischen zum Standard geworden, für die Betroffenen bringt der Eingriff einen enormen Zugewinn an Lebensqualität mit sich. Mit dem verbreiteten Einsatz der Endoprothesen kommt es aber immer häufiger auch zu allergischen Reaktionen auf einen der Implantatwerkstoffe.}

\begin{abstract}
mplantate ersetzen im menschlichen Körper nicht nur Gelenke. Sie kommen auch im Hals-Nasen-Ohren-Bereich und im dentalen Bereich oder als Stents zum Einsatz. Neben guten mechanischen Eigenschaften müssen die dabei verwendeten Materialen eine hohe Bioverträglichkeit aufweisen. Denn, so erklärte Priv.-Doz. Dr. Peter Thomas von der Ludwig-Maximilians-Universität, München, jedes Einsetzen von Implantatmaterialien führe nicht nur durch das Operationstrauma, sondern auch durch die Reaktion auf das Fremdmaterial zu einer Entzündungsantwort des Körpers. Wie stark diese ausfällt und wie lange sie andauert, hängt von der Lokalisation des Implantats und den Materialeigenschaften ab. Hier spielen beispielsweise die Freisetzung von Metallionen sowie Art und Menge der Partikelbildung eine Rolle. Einfluss hat jedoch auch die individuelle immunologische Reaktionsbereitschaft des betroffenen Organismus.
\end{abstract}

\section{Beschwerden bis zur Implantat- lockerung}

Wenn eine spezifische Sensibilisierung von Lymphozyten stattfindet oder eine Allergie bereits besteht, können Überempfindlichkeitsreaktionen gegen Implantatbestandteile auftreten. Als Folge einer allergischen Reaktion vom Typ IV sind dann lokale oder ausgedehnte Ekzeme, Wundheilungsstörungen oder eine sterile Osteomyelitis möglich. „Ob auch eine Implantatlockerung allergiebedingt entstehen kann, wird noch kontrovers diskutiert“, ergänzte Thomas. „Monozyten und Lymphozyten können dabei in-

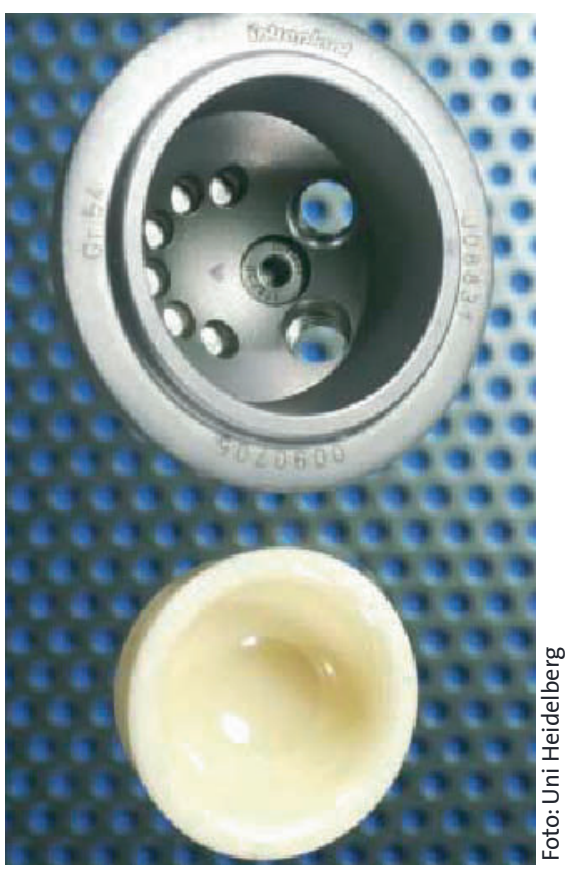

Allein im Jahr 2003 wurden in Deutschland 12.000 Hüft- und 2.300 Knieendoprothesen eingesetzt.

dividuell unterschiedliche Apoptoseschwellen haben", erläutert Thomas. Es kann also sein, dass der Kontakt mit typischen Kontaktallergenen wie Chrom, Nickel oder Kobalt entweder zu einer Apoptose führt oder aber eine Immunantwort der Lymphozyten erfolgt.

Unter welchen Bedingungen bei Patienten, die bereits gegen Metalle sensibilisiert sind, das Einbringen des relevanten Metalls tatsächlich zu Komplikationen führt, ist noch weitgehend unklar. Bis zu $12 \%$ der Bevölkerung seien gegen Nickel, bis zu $5 \%$ gegen Kobalt und Chrom sensibilisiert, erklär- te Thomas. Doch seien bisher nur wenige Patienten mit einer Allergie gegen Implantate, die Edelstahl oder KobaltChrom-Legierungen enthalten, entdeckt worden. Genaue Angaben über die Häufigkeit von Allergien gegen Implantatwerkstoffe gibt es noch nicht.

\section{Was tun bei Verdacht auf Implantatallergie?}

Bei Verdacht auf eine Implantatüberempfindlichkeit ist zunächst ein Epikutantest angezeigt. Der hilft jedoch nur bedingt weiter. Denn derzeit ist es lediglich in Einzelfällen möglich, eine kausale Verknüpfung zwischen der Unverträglichkeit eines Implantats und einer im Hauttest gefundenen Kontaktallergie auf Implantatkomponenten herzustellen. „Bei verblüffend vielen Personen, die eigentlich eine Nickelallergie haben und denen aus Unkenntnis dieses Umstands nickelhaltiges Material eingesetzt wird, zeigen sich keineswegs sichtbare Komplikationen", berichtete Thomas. Bei einem Teil der Patienten mit komplikationsbedingten Revisionsoperationen, die keine kutanen Symptome aufwiesen, habe man aber dennoch periimplantäre entzündliche Infiltrate gefunden, die Charakteristika von Typ-IV-Reaktionen aufwiesen.

Es sei weiter möglich, so Thomas, im Gewebe Aktivierungsmarker und die Zytokinexpression mit der PolymeraseKettenreaktion zu analysieren. Damit lässt sich etwa ein TH1-typisches Entzündungsmuster identifizieren. Darüber hinaus versucht man periimplantäre $T$ Lymphozyten funktionell zu charakterisieren. Dieser Ansatz ist jedoch sehr aufwendig und kann zum jetzigen Zeitpunkt noch nicht in die Routinediagnostik übernommen werden.

So bleibt dem Arzt, so Thomas' Fazit, letztlich nur die Möglichkeit, bei der Diagnostik Detektivarbeit zu leisten und das klinische Gesamtbild zu bewerten. Wenn sich der Verdacht auf eine Implantatallergie erhärtet, sollte das Fremdmaterial entfernt werden. Verschwinden die Beschwerden, ist dies die letzte Bestätigung der Diagnose. $\quad b m$

Thomas P. Implantatüberempfindlichkeit. Allergiekongress Aachen 2004, Aachen, 15.-19. September 2004 